\title{
No evidence for an association between extreme longevity and Microsomal Transfer Protein polymorphisms in a longitudinal study of 1651 nonagenarians
}

\author{
Lise Bathum ${ }^{* 1,2}$, Lene Christiansen ${ }^{1,2}$, Qihua Tan ${ }^{1,2}$, James Vaupel $^{2,3}$, Bernard Jeune ${ }^{2}$ \\ and Kaare Christensen ${ }^{2}$ \\ ${ }^{1}$ Department of Clinical Biochemistry, Odense University Hospital, Odense, Denmark; ${ }^{2}$ Department of Epidemiology, \\ University of Southern Denmark, Denmark; ${ }^{3}$ The Max Planck Institute for Demographic Research, Rostock, Germany
}

Previous studies have reported two SNPs and a haplotype marker within the Microsomal Transfer Protein gene associated with extreme longevity. Here, we test this finding in a longitudinal study of nonagenarians and in an association study. Participants in the Danish 1905 cohort study (1651 participants aged 92-93 years) were genotyped for the two SNPs (rs2866164 and Q95H) in the Microsomal Transfer Protein gene recently reported to be associated with longevity. The 1905 Cohort has been followed for 6.5 years, during which period $\mathbf{8 3} \%$ of the cohort has died. Furthermore, a group of 575 middle-aged Danish twins (mean age 53.7 years) were tested as a younger control group. The risk haplotype had no significant survival disadvantage ( $P$-values: $0.56,0.31$ and 0.97 in the total population of nonagenarians, males and females, respectively) after 6.5 years of follow-up. The distributions of the suggested risk alleles (rs2866164-G and Q95) and the resulting haplotypes are very similar and not statistically different between the two age cohorts. The frequency for rs2866164-G is in the middle-aged compared to the nonagenarians 25.4 and $23.6 \%$ in males and 23.0 and $26.1 \%$ in females. The frequency for the risk haplotype is in the middle-aged compared to the nonagenarians 22.7 and $19.2 \%$ in males and 18.1 and $21.8 \%$ in females. In conclusion, our longitudinal study of survival in the 10th decade of life and an association study in a genetically homogeneous population provided no support for an association between the Microsomal Transfer Protein gene and extreme longevity.

European Journal of Human Genetics (2005) 13, 1154-1158. doi:10.1038/sj.ejhg.5201468;

published online 13 July 2005

Keywords: microsomal transfer protein; ageing; longevity

\section{Introduction}

Major efforts have been made for identifying genes involved in ageing. An increasing number of studies are

*Correspondence: Dr L Bathum, Department of Clinical Biochemistry, Odense University Hospital, Sdr. Boulevard 29, DK-5000 Odense C, Denmark. Tel: + 456541 2873; Fax: + 456541 1911;

E-mail: L.Bathum@ouh.fyns-amt.dk

Received 27 October 2004; revised 9 June 2005; accepted 14 June 2005; published online 13 July 2005 reporting genes influencing human longevity including cytochrome P450 genes, mitochondrial DNA and genes involved in lipoprotein metabolism and coagulation. ${ }^{1}$ However, only the Apolipoprotein E gene has consistently been associated with age-related changes in frequency. ${ }^{2-4}$ Recent studies performed a genome-wide linkage scan in long-lived individuals and provided evidence for a locus for longevity at chromosome 4 near microsatellite marker D4S1564. ${ }^{5,6}$ Fine mapping of the region identified Microsomal Triglyceride Transfer Protein (MTP) as the gene 
responsible for the chromosome 4 linkage peak, ${ }^{7}$ although a confirmatory study in a French population did not confirm this observation. ${ }^{7}$ The two SNPs found to account for the majority of the variation at the locus were rs2866164-a SNP in complete linkage disequilibrium with rs1800591 $(-493 \mathrm{G} / \mathrm{T})$, an MTP promoter mutation- and $M T P \mathrm{Q} / \mathrm{H}$ - a semiconservative mutation in exon 3 of MTP (glutamine to histidine at aminoacid 95). ${ }^{7}$ The rs2866164-G allele and the haplotype composed of rs2866164-G/MTP-Q95 were found to be significantly underrepresented in long-lived individuals compared to a group of younger controls.

MTP is essential for the assembly of apolipoprotein B-containing lipoproteins by the liver and small intestine ${ }^{8}$ and lack of the protein leads to abetalipoproteinemia, a rare genetic disorder characterized as an inability to produce chylomicrons and very low-density lipoproteins. MTP is a good candidate for a gene involved in human longevity as its functions and critical position in lipoprotein assembly resembles Apolipoprotein E, the most extensively studied and consistently reproduced gene affecting human longevity. ${ }^{3,4}$ Lipid-lowering therapy is established as a proven intervention to reduce atherosclerosis and its complications. MTP is an investigational target for a new generation of lipid-lowering drugs and has reached trials in animal models. ${ }^{9}$

It is important to point out that the finding by Geesaman et al (2003) was not confirmed in the French data reported in the same paper. Given this discrepancy, there is a need for independent studies to confirm the reported association between MTP and exceptional longevity using large and well-defined samples and preferable longitudinal studies. In the Danish 1905 cohort study, we assessed the complete Danish 1905 cohort in 1998. ${ }^{10,11}$ In total, 2262 participated in the survey, which included an interview, physical and cognitive tests, as well as collection of biological material (1651 participants provided a DNA sample). Hence, they were 92-93 years when they entered the study and have been followed regarding mortality until January 2005. We examined the two reported SNPs in the complete Danish 1905 cohort study. If MTP does infact play a role in human longevity, a longitudinal study of nonagenarians - a population with a very high mortality and 6.5 years of follow-up - should be able to detect a mortality difference. To rule out that a selection has taken place before the age of 92 years, a younger control group of 575 middle-aged Danish twins (mean age 53.7 years) were included.

\section{Methods}

The participants were selected from two major surveys conducted by our group, the 1905 cohort, all Danes born in 1905 (1651 DNA samples) ${ }^{10}$ ascertained in 1998 when they were 92-93 years and the study of Middle-Aged Danish twins (MADT), a random sample of twin pairs born between 1931 and 1952 (4171 DNA samples). ${ }^{12}$ These studies comprised a home-based 2-h multidimensional interview and the sampling of DNA by means of a finger prick or a cheek swab. All samples from the 1905 cohort $(28.2 \%$ males) were included and 575 samples (mean age 53.7 years, age range 45-67 years, $47.9 \%$ males) - only one from each twin pair-were chosen from MADT.

\section{Genotyping}

DNA was isolated from cheek swabs and blood spots with the use of QIAamp ${ }^{\circledR}$ DNA Mini Kit (Qiagen, Germany). The Taqman technology was used to genotype for the two polymorphisms related to MTP. Primers and probes were designed using Primer Express ${ }^{\circledR}$ software (Applied Biosystems). Primers and probes used were rs2866164: sense 5'-GTTTAAACAAGAAATCTCAAACCATATCTAC-3', antisense $5^{\prime}$-CTTGTAAATATAGTATATTTACCATATCTTAGT AAGTAAA-3', C-probe 5'-FAM-CTGTTTACCAGACTGCTT, G-probe 5'-VIC-CTGTTTAGCAGACTGCT and MTP Q/H: sense 5'-CTTTCTGTTACTCCAGATGAAGGATGT-3', antisense 5'-TTTCCCATTATTTTAGATGGGCTTT-3', Q-probe 5'-FAM-CTCTCCTCTCTGCTGATT, H-probe 5'-VIC-CT CTCCTCTGTGCTGATT. All probes contain minor groove binder groups and a nonflourescent quencher attached to their $3^{\prime}$ ends. The real-time analyses were performed at the ABI PRISM 7700 Sequence Detection System equipped with the allelic discrimination module (software version 1.7; Applied Biosystems). DNA was amplified in a total volume of $10 \mu \mathrm{l}$ containing $5 \mu \mathrm{l}$ Taqman Universal Master Mix (Applied Biosystems), $900 \mathrm{~nm}$ of each primer (DNA Technology), $200 \mathrm{~nm}$ of each probe (Applied Biosystems) and $\sim 10 \mathrm{ng}$ DNA at an annealing temperature of $60^{\circ} \mathrm{C}$ and 40 PCR cycles. In total, 26 subjects from the 1905 cohort and 24 of the middle-aged twins could not be genotyped for one or both polymorphisms due to a poor quality of DNA. Only subjects that could be genotyped for both polymorphisms are used in the statistical analysis. Four subjects gave unclear genotyping results for $\mathrm{H} 95 \mathrm{Q}$ and exon three in the MTP gene was sequenced. A missense mutation under the probe, E98D, was found in these samples. Only three of the expected four haplotypes were seen as expected, since the rs2866164-C/H95 haplotype has not previously been detected.

\section{Statistical analyses}

The participants were followed from the date of blood sampling and until emigration, death or end of study period (January 2005). Information on emigration or death was retrieved from the Danish Central Population Register, which is continuously updated. The Kaplan-Meier method was used to plot cumulative survival curves. For the survival analysis, the subjects were stratified by the 
occurrence of the suggested risk haplotype composed of the rs2866164-G and the Q95 allele.

The difference in distribution of the risk alleles/haplotype (rs2866164-G, MTP-Q95 and the haplotype composed of rs2866164-G/MTP-Q95) between the two age cohorts was tested with a critical ratio $(Z)$ test, which calculates a 95\% confidence interval of the difference between the two populations and a two-sided $P$-value. ${ }^{13,14}$ Stata version 8 (Stata Corporation) and EpiCalc 2000 (version 1.02; Gilmann and Myatt, Brixton, England) were used for statistical analysis.

\section{Results}

The Kaplan-Meier cumulative survival curves for the nonagenarian males and females are shown in Figures 1 and 2. Age is not a confounding factor as all participants are born in 1905. There is no significant survival difference, as tested by the logrank test, despite the large sample size and although more than $83 \%$ deceased at the end of the follow-up period; the $P$-values are $0.56,0.31$ and 0.97 in the total population of nonagenarians, males and females, respectively.

The genotyping results are shown in Tables 1-3. The distribution is in Hardy-Weinberg equilibrium for both SNPs and haplotypes in both age groups $(P \geq 0.29)$. None of the prevalence differences of the suggested risk alleles (rs2866164-G and MTP Q95) and the combined risk haplotype is significant on a 0.05 level (Tables 1-3). Furthermore, there is no statistically different distribution between the subjects from the 1905 cohort alive at the last follow-up in January 2005 (6.5 years after recruitment) and those who died before January 2005.

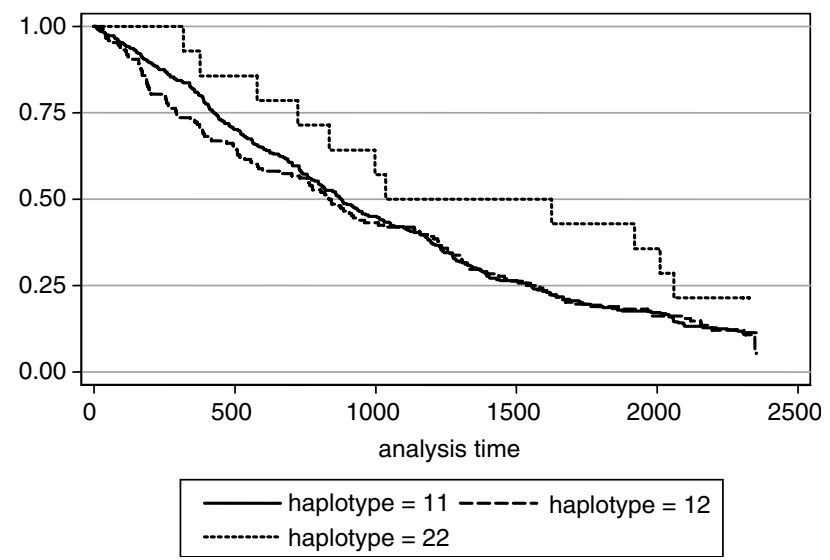

Figure 1 Kaplan-Meier survival estimates in males from the 1905 cohort study $(N=457)$. Haplotype 2 is the suggested risk haplotype composed of the rs2866164-G and the Q95 allele and haplotype 1 is all other haplotypes. The analysis time is days. The difference is not significant $(P=0.31)$.

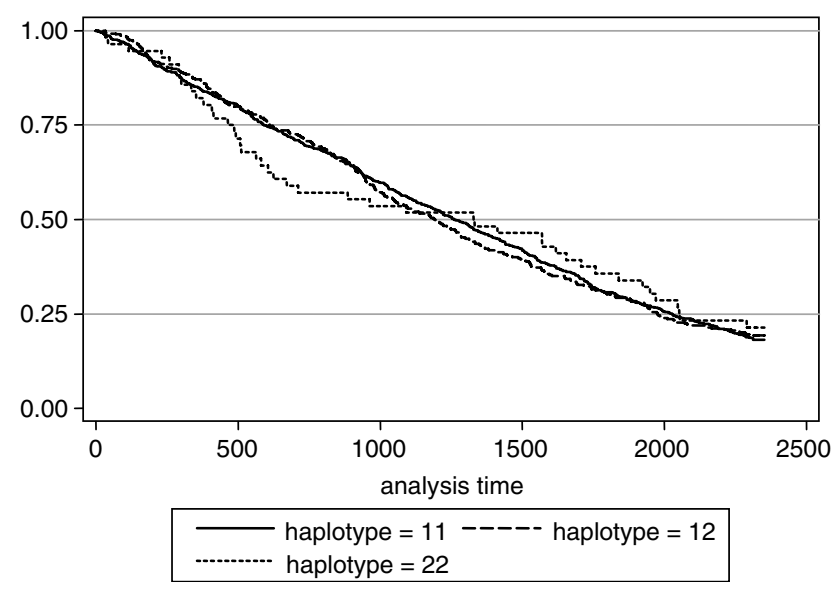

Figure 2 Kaplan-Meier survival estimates in females from the 1905 cohort study $(N=1168)$. Haplotype 2 is the suggested risk haplotype composed of the rs2866164-G and the Q95 allele and haplotype 1 is all other haplotypes. The analysis time is days. The difference is not significant $(P=0.97)$.

Table 1 Risk SNP allele counts in males

\begin{tabular}{lccr}
\hline SNP & Middle-aged & 1905-cohort & Survivors 1905 \\
\hline rs 2866164-C & $394(74.6 \%)$ & $698(76.4 \%)$ & $82(77.4 \%)$ \\
rs 2866164-C & $134(25.4 \%)$ & $216(23.6 \%)$ & $24(22.6 \%)$ \\
MTP Q95 & $515(97.5 \%)$ & $874(95.6 \%)$ & $104(98.1 \%)$ \\
MTP H95 & $13(2.5 \%)$ & $40(4.4 \%)$ & $2(1.9 \%)$ \\
\hline
\end{tabular}

Shown are counts (frequencies) for rs2866164 and MTP Q/H.

Table 2 Risk SNP allele counts in females

\begin{tabular}{lccc}
\hline SNP & Middle-aged & 1905-cohort & Survivors 1905 \\
\hline rs 2866164-C & $442(77.0 \%)$ & $1727(73.9 \%)$ & $322(72.9 \%)$ \\
rs 2866164-C & $132(23.0 \%)$ & $609(26.1 \%)$ & $120(27.1 \%)$ \\
MTP Q95 & $546(95.1 \%)$ & $2236(95.7 \%)$ & $423(95.7 \%)$ \\
MTP H95 & $28(4.9 \%)$ & $100(4.3 \%)$ & $19(4.3 \%)$ \\
\hline
\end{tabular}

Shown are counts (frequencies) for rs2866164 and MTP Q/H.

\section{Discussion}

In contrast with the previous publication in PNAS by Geesaman et al, ${ }^{7}$ our data fail to provide evidence for a role for MTP in human longevity. There is no increased mortality among carriers of the 'risk haplotype' composed of the rs2866164-G and the Q95 allele in the longitudinal study of 1651 nonagenarians and, furthermore, there is no significant difference between the distribution of the two SNPs and the 'risk haplotype' in the two tested age cohorts. The paper by Geesaman et $a l^{7}{ }^{7}$ included a confirmatory study of a French centenarian cohort compared to younger French controls. There was no significant difference in allele frequencies with respect to the risk haplotype. However, the risk haplotype was found to be out of 
Table 3 Haplotype counts and frequencies

\begin{tabular}{|c|c|c|c|c|c|c|}
\hline \multirow[b]{2}{*}{ Allele } & \multicolumn{2}{|c|}{ Middle-aged } & \multicolumn{2}{|c|}{1905 cohort } & \multicolumn{2}{|c|}{ Survivors 1905} \\
\hline & Rs2866164-C & rs2866164-G & rs2866164-C & rs2866164-G & rs2866164-C & rs2866164-G \\
\hline Males & \multicolumn{2}{|c|}{ Mean age: 55.5 years } & \multicolumn{2}{|c|}{ Age $92-93$ years } & \multicolumn{2}{|c|}{ Age 99 years } \\
\hline $\begin{array}{l}\text { Q95 } \\
\text { H95 }\end{array}$ & $\begin{array}{c}394(74.6 \%) \\
0\end{array}$ & $\begin{array}{c}120(22.7 \%) \\
14(2.7 \%)\end{array}$ & $\begin{array}{c}698(76.4 \%) \\
0\end{array}$ & $\begin{array}{c}176(19.2 \%) \\
40(4.4 \%)\end{array}$ & $\begin{array}{c}82(77.4 \%) \\
0\end{array}$ & $\begin{array}{c}22(20.8 \%) \\
2(1.9 \%)\end{array}$ \\
\hline Females & \multicolumn{2}{|c|}{ Mean age: 52.1 years } & \multicolumn{2}{|c|}{ Age $92-93$ years } & \multicolumn{2}{|c|}{ Age 99 years } \\
\hline $\begin{array}{l}\text { Q95 } \\
\text { H95 }\end{array}$ & $\begin{array}{c}442(77.0 \%) \\
0\end{array}$ & $\begin{array}{c}104(18.1 \%) \\
28(4.9 \%)\end{array}$ & $\begin{array}{c}1727(73.9 \%) \\
0\end{array}$ & $\begin{array}{l}509(21.8 \%) \\
100(4.3 \%)\end{array}$ & $\begin{array}{c}322(72.9 \%) \\
0\end{array}$ & $\begin{array}{c}101(22.9 \%) \\
19(4.3 \%)\end{array}$ \\
\hline
\end{tabular}

Shown are counts (frequencies) for the two-SNP haplotype formed by combining alleles of the two SNP's in MADT and the 1905 cohort. The 1905 survivors are the participants still alive January 2005. Only three of the four haplotypes were observed.

Hardy-Weinberg equilibrium with the other haplotypes in the centenarians, but not in the controls. We find frequencies in our middle-aged population very close to the French control group - the frequency is $24.1 \%$ for rs $2866164-\mathrm{G}$ compared to $26.4 \%$ in the French population and for the 'risk haplotype' the frequency is $20.1 \%$ compared to $19.6 \%$. The risk haplotype is in HardyWeinberg equilibrium in our cohorts for both the individual SNPs and the combined haplotypes.

One explanation for the discrepancy between the study by Geesaman et $a l^{7}$ and our data could be that the American population is less homogeneous than ours. A major problem in genetic association studies is the failure to adequately match the genetic backgrounds of the cases and controls, and in the study of Geesaman et al, the cases and controls were born more than 50 years apart. The Danish population is known to be genetically and ethnically very homogeneous and therefore well suited for association studies in different age groups. The middleaged twins were born and grew up in Denmark. Very little immigration has taken place in Denmark in the years between 1905 and 1960. The minimal immigration into Denmark in the cohorts comprised by these surveys, the high response rate in both surveys (63\% in the 1905 cohort study and $80 \%$ in the middle-aged twins) and the high genetic homogeneity in the Danish population, minimize population stratification as well as selection bias within the population. Furthermore, we have the ability to conduct a longitudinal study on nonagenarians as we have followed the 1905 cohort for 6.5 years, which is not affected by any population stratification. And the results all point in the same direction - no haplotype-dependent mortality difference in the 1905 cohort and no significant difference in frequency of the tested SNPs between the middle-aged population and the 1905 cohort. However, although the Danish population is very homogeneous, no cross-generational comparisons are necessarily immune from stratification and it is therefore possible-but very unlikely - that our results are false-negative due to stratification.
Our population is slightly younger than that of Geesaman et al. ${ }^{7}$ Their long-lived individuals had a mean age of 100.8 years. Participants in our study population were 92-93 years when they entered the study and 99 years at the end of follow-up - and intuitively this seems to be an already much selected population, close to becoming centenarians. However, being $92-93$ is only 'half way to becoming a centenarian' in terms of selection: only about one in 20 of the 1905 cohort made it of age $92-93$, but it is also only about one in 20 of these survivors to age 92-93 who will celebrate a 100-year birthday. Hence, the Danish 1905 cohort provides a powerful opportunity to test for selection against a genotype marker and even though our cases are slightly younger, this large study of 1651 subjects aged 92-93 years should be able to detect a significant difference. As can be seen from Figures 1 and 2, there is no significant survival difference between those carrying the suggested risk allele and those without this allele. In males there is even a small-not significant-tendency to a better survival of those homozygous for the suggested risk allele.

The finding of the linkage to exceptional longevity at chromosome 4 near the MTP gene was the result of a genome-wide scan in 308 extremely long-lived individuals in 137 sib ships with the use of non-parametric linkage analysis. ${ }^{5}$ Longevity is a complex trait and is probably modulated by multiple genes. Our group has previously shown that mapping a rare dominant genetic variation that reduces hazard of death by half with the use of nonparametric linkage analysis would require a sample of more than 600 long-lived pairs ${ }^{15}$ to ensure acceptable power. In the case of localization of recessive genes, the power is larger and the method is able to localize recessive genes with relatively small effects with the same sample size (600 pairs). So although the power issue may depend heavily on the true genetic nature in maintaining survival, the results from small-scale sib-pair investigations, such as the study of Puca et al, ${ }^{5}$ should be interpreted with caution and should be subject to independent replications. Statis- 
tical evidence for an association between an allele and a phenotype theoretically comes from three different situations, the allele itself might be functional, the allele may be correlated with a causative gene nearby or the association is attributable to chance. ${ }^{16}$ Population stratification can induce important bias, but the original study by Geesaman et $\mathrm{al}^{7}$ used proactive matching of cases and controls to protect against stratification-induced false positives, so it is unlikely that population stratification is in fact the source of the discrepancy. The results from Geesaman could be caused by random chance - simply a false-positive fluctuation-although true population genetic differences or gene-environment differences between the two populations cannot be ruled out.

To conclude, a previous study ${ }^{7}$ reported a haplotype marker within the MTP gene as a modifier of human lifespan, but our confirmatory study does not support an association between extreme longevity and the Microsomal Transfer Protein. Replication studies are crucial to cope with the false positive results in mapping human complex trait genes. The fact that our results, based on a large and well-defined sample, fail to confirm the association between the MTP gene and longevity reported by Geesaman et al illustrates the necessity of replication studies in mapping genes which affect human longevity.

\section{Acknowledgements}

This work was supported by the US National Institute on Aging Research Grant NIA-PO1-AG08761, The Danish National Research Foundation.

\section{References}

1 De Benedictis G, Tan Q, Jeune B et al: Recent advances in human gene-longevity association studies. Mech Ageing Dev 2001; 122: 909-920.
2 Gerdes LU, Jeune B, Ranberg KA, Nybo H, Vaupel JW: Estimation of apolipoprotein E genotype-specific relative mortality from the distribution of genotypes in centenarians and middle-aged men: apolipoprotein E gene is a 'frailty gene', not a 'longevity gene'. Genet Epidemiol 2000; 19: 202-210.

3 Tan Q, Christiansen L, Christensen K, Kruse TA, Bathum L: Apolipoprotein E genotype frequency patterns in aged Danes as revealed by logistic regression models. Eur J Epidemiol 2004; 19: $651-656$.

4 Ewbank DC: Mortality differences by APOE genotype estimated from demographic synthesis. Genet Epidemiol 2002; 22: 146-155.

5 Puca AA, Daly MJ, Brewster SJ et al: A genome-wide scan for linkage to human exceptional longevity identifies a locus on chromosome 4. Proc Natl Acad Sci USA 2001; 98: 10505-10508.

6 Reed T, Dick DM, Uniacke SK, Foroud T, Nichols WC: Genomewide scan for a healthy aging phenotype provides support for a locus near D4S1564 promoting healthy aging. J Gerontol A Biol Sci Med Sci 2004; 59: 227-232.

7 Geesaman BJ, Benson E, Brewster SJ et al: Haplotype-based identification of a microsomal transfer protein marker associated with the human lifespan. Proc Natl Acad Sci USA 2003; 100: 14115-14120.

8 Swift LL, Zhu MY, Kakkad B et al: Subcellular localization of microsomal triglyceride transfer protein. I Lipid Res 2003; 44: $1841-1849$.

9 Wierzbicki AS: New lipid-lowering agents. Expert Opin Emerg Drugs 2003; 8: 365-376.

10 Nybo H, Gaist D, Jeune B et al: The Danish 1905 cohort: A genetic-epidemiological nationwide survey. Age Age 2001; 13: $32-46$.

11 Nybo H, Petersen HC, Gaist D et al: Predictors of Mortality in 2249 Nonagenarians - The Danish 1905-Cohort Survey. JAGS 2003; 51: $1365-1373$.

12 Gaist D, Bathum L, Skytthe A et al: Strenght and anthropometric measures in identical and fraternal twins: no evidence of masculinization of females with male co-twins. Epidemiology 2000; 11: 340-343.

13 Fleiss J: Statistical Methods for Rates and Proportions, 2nd edn. New York: John Wiley \& Sons, 1981.

14 Cooke D, Craven AH, Clarke G: Basic Statistical Computing, 2nd edn, London: Edward Arnold, 1990.

15 Tan Q, Zhao JH, Iachine I et al: Power of non-parametric linkage analysis in mapping genes contributing to human longevity in long-lived sib-pairs. Genetic Epidemiology 2004; 26: 245-253.

16 Cardon LR, Palmer LJ: Population stratification and spurious allelic association. Lancet 2003; 361: 598-604. 